\title{
A Rare Case of Intravenous Pesticide Poisoning with Upper Extremity Necrosis
}

\author{
Jae Doo Joo, Dong Hee Kang, Hyonsurk Kim \\ Department of Plastic and Reconstructive Surgery, Dankook University Hospital, Cheonan, Korea
}

\begin{abstract}
Intentional pesticide poisoning is not uncommonly encountered in emergency care settings, and the route of administration is generally oral. We present a peculiar case of intravenous pesticide injection and the prolonged management of upper extremity necrosis that ensued. A man who had injected an unidentified pesticide into his antecubital vein was admitted for acute renal failure and left arm cellulitis. After vital status stabilization, the patient was referred for cellulitis management. A febrile inflammatory draining wound was centered on the antecubital fossa. Incisional drainage and fasciotomy revealed thick pesticide-smelling mucoserous fluid and liquifaction necrosis following the course of thrombosed and sclerotic vessels. Serial surgical debridement and negative pressure wound therapy allowed local skin flap coverage. However, a large post-discharge seroma developed and the patient was re-admitted for nearly a month of additional wound management which finally achieved successful recovery with full range of motion of the arm and hand. Intravenously injected pesticide is poorly cleared by local tissues and can cause abscess formation and liquifactory necrosis. Recognition of the poisoning agent and early initiation of fasciotomy and serial debridement is necessary for successful wound management of the involved extremity.
\end{abstract}

Keywords: Pesticide, Poisoning, Intravenous injection, Upper extremity

\section{Introduction}

Pesticides can be classified as chlorinated hydrocarbons, acetyl cholinesterase inhibitors and botanical agents. Most pesticides exert their efficacy by poisoning the nervous system in insects and produce systemic toxicity including neural manifestations similarly in humans [1]. Although the vast majority of pesticide poisonings are accidental, absorbed through the skin or respiratory tract, intentional pesticide poisoning is unfortunately not an uncommon form of substance abuse in emergency care settings, and the route of administration is generally oral ingestion. Compared to ingestion or inhalation, injection of pesticides (whether subcutaneous, intravenous or intramuscular) is a relatively rare event [2]. Besides the systemic effects similar to enteral ingestion, parenteral pesticide injection can cause local tissue damage ranging from inflammation and cellulitis to abscess formation and even compartment syndrome. Injected pesticides tend to be poorly cleared or detoxified by local tissues, can cause liquefaction necrosis, and have inherent systemic effects such as chemical or hemorrhagic pneumonitis if sufficient quantity enters the circulation; therefore the injection wound must be intensively managed as well as the patient's systemic condition. Some cases of pesticide injection have been reported, mainly focusing on systemic treatment without long term follow up [1,2,7]. We present a peculiar case of attempted suicide by intravenous pesticide injection and the prolonged wound management of upper extremity necrosis that followed.

\begin{tabular}{l} 
Case Report \\
\hline Received: May 9, 2018 \\
Revised: June 22, 2018 \\
Accepted: June 22, 2018 \\
Corresponding author: \\
Hyonsurk Kim, M.D. \\
Department of Plastic and Reconstructive \\
Surgery, Dankook University Hospital, 201 \\
Manghates \\
Korea \\
Tel: +82-41-550-6285 \\
Fax +82-41-556-0524 \\
E-mail: kruezel@gmail.com \\
This article was presented at The Wound Meeting \\
2018, in Seoul, Korea, March 30, 2018. \\
This is an Open Access article distributed under the terms \\
of the Creative Commons Attribution Non-Commercial \\
License (http://creativecommons.org/licenses/by-nc/4.0/) \\
which permits unrestricted non-commercial use, \\
distribution, and reproduction in any medium, provided the \\
original work is properly cited. \\
o 2018 Korean Wound Management Society
\end{tabular}

Received: May 9, 2018

Revised: June 22, 2018

Accepted: June 22, 2018

Corresponding author:

Hyonsurk Kim, M.D. Korea

Tel: $+82-41-550-6285$

Fax +82-41-556-0524

E-mail: kruezel@gmail.com

This article was presented at The Wound Meeting 2018, in Seoul, Korea, March 30, 2018.

the terms the Creative Commons Attribution Non-Commercial (http.//creativecommons.org/licenses/by-nc/4.0/) distribution, and reproduction in any medium, provided the (c) 2018 Korean Wound Management Society 


\section{Case report}

A 52-year-old male arrived at the emergency department with decreased mentality. The patient, who had served as a corpsman during military service and had no past medical history, apparently had injected pesticide into his left antecubital vein for suicidal purposes. His initial Glasgow scale was $11 / 15$ and it was impossible to determine the amount and type of the pesticide. A small wound was observed in the patient's left antecubital fossa, with swelling, erythema and pain. After intubation, the patient was admitted to intensive care for acute renal failure, hyperkalemia and left arm cellulitis, where empirical antibiotics were administered for wound care. After continuous renal replacement therapy stabilized his blood chemistry, the patient was referred to Plastic Surgery for left arm cellulitis management, 9 days after admission. No pulmonary or other systemic complications were noted during this period. The patient was still delirious, and upper extremity range of motion and sensation were hard to determine. Febrile inflammation with a draining wound was centered on the left antecubital fossa, and bedside incisional drainage released turbid fluid and a heavy organophosphate-like odor (Fig. 1). Cultures obtained from the draining fluid disclosed Pseudomonas aeruginosa growth. Intravenous antibiotics (Meropen, Meropenem, Yuhan Co., Seoul, Korea) were administered and a decision was made for surgical exploration with debridement. Under general anesthesia, an incision extending $5 \mathrm{~cm}$ proximal and distal from the antecubital wound was made. The supra-fascial layer displayed widespread pesticide-smelling pink-brownish fluid, and fasciotomy revealed more pesticidesmelling mucoserous fluid and liquifaction necrosis of muscle following the course of thrombosed and sclerotic vessels (Fig.

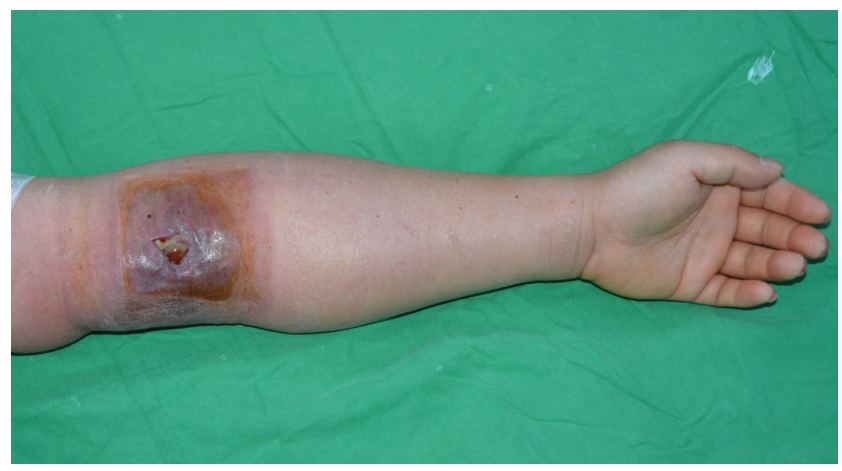

Fig. 1. Preoperative clinical view. Erythematous swelling, pain, tenderness and febrile sense centered on the antecubital draining wound.
2). The sclerotized brachial vein was ligated and excised. Liquified tissues were debrided and necrotic fluid thoroughly flushed with copious saline irrigation, followed by application of negative pressure wound therapy (NPWT). Two days later, a strong odor of pesticide still permeated the surgical site, and through an additional transverse incision more necrotic tissue and vessels were resected (Fig. 3A). Another three days later, the third serial surgical debridement and NPWT revealed no remnant pesticide odor and tissue cultures were also negative (Fig. 3B). On day 8 after the initial surgery clinical inflammatory symptoms (heating sense, erythema, swelling and tenderness) were resolved and granulation tissue was growing over the clean wound bed (Fig. 3C). Follow-up wound cultures remained negative. The wound was subsequently closed with local skin flaps on the twelfth day (Fig. 3D), and the patient was discharged with a fully healed wound 15 days later. Intravenous antibiotics were discontinued one day before discharge. However, just 2 days after discharge, a large seroma developed under the skin flaps with symptoms of swelling, pain and tenderness. A small opening with skin breakdown had also appeared over the seroma site (Fig. 4). Seroma cultures showed no bacterial growth. The patient was re-admitted for additional wound management; nearly a month of NPWT, revisional repair and compressive dressings allowed complete wound healing with full range of motion of the arm and hand. The same regimen of antibiotics was re-administered for 20 days during hospitalization. At 6 months follow-up, hand grip strength was not fully recovered yet but sufficient enough to not cause any discomfort in daily activities (Fig. 5). Wound site sensory had completely recovered, as had forearm sensation except for a $5-\mathrm{cm}$ area on the ulnar aspect.

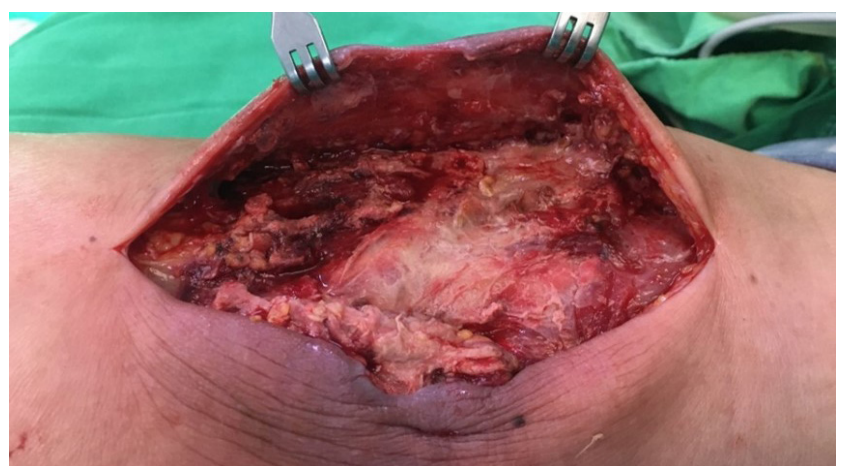

Fig. 2. Intraoperative view, initial surgery. Surgical exploration revealed a large amount of mucoserous fluid with a heavy organophosphate-like odor and liquifaction necrosis of muscle along thrombosed necrotic vessels. 

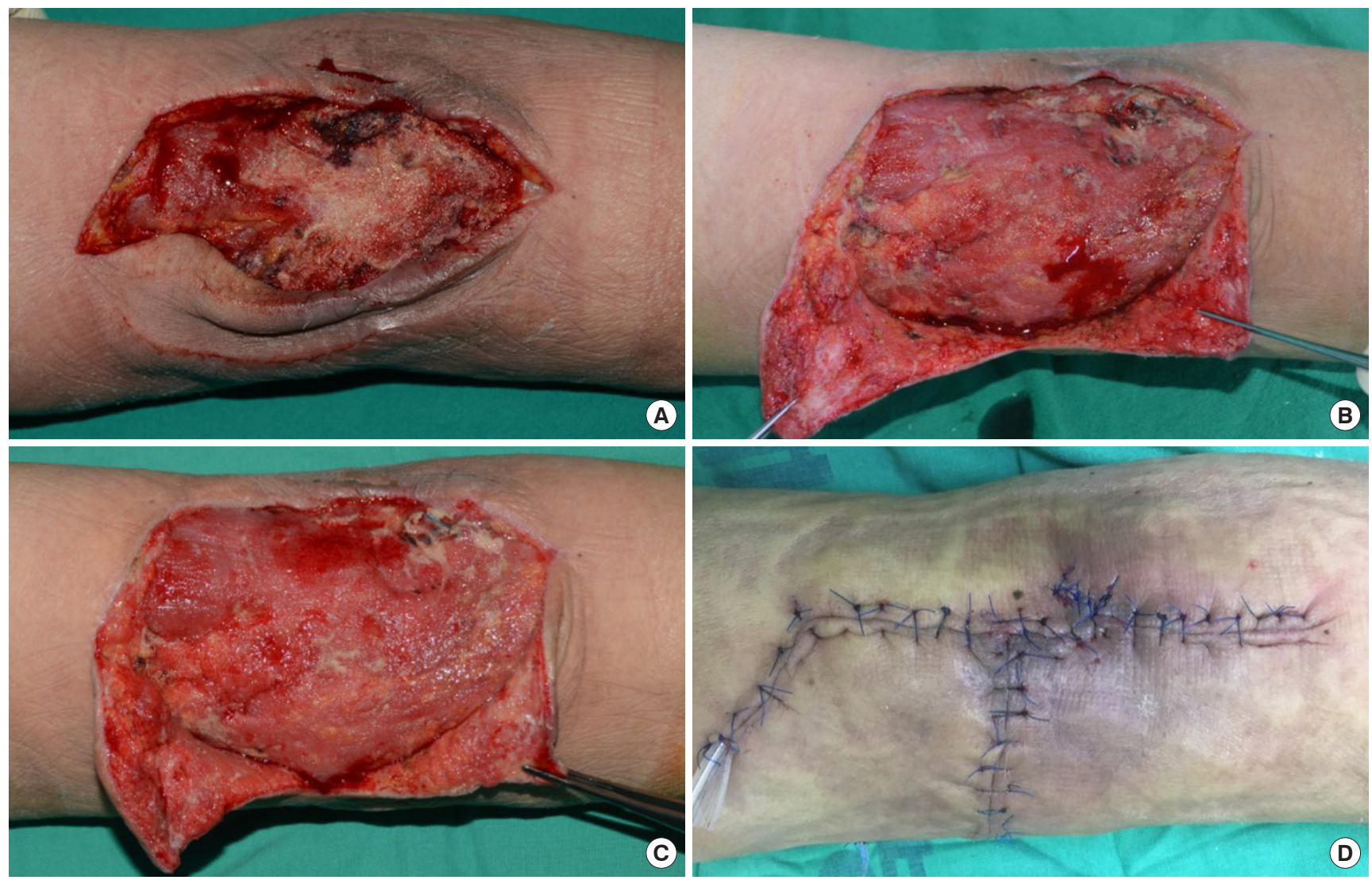

Fig. 3. Serial clinical photographs. (A) Preoperative photo of the second surgical debridement. Necrotic tissue with mucoserous discharge remained after initial debridement and NPWT. A strong odor of the pesticide still permeated the operating room. (B) Preoperative photo of the third surgical debridement. There was no residual odor after 5 days, and granulation tissue formation can be seen. (C) Clinical photo of the eighth day after initial surgery. Clinical symptoms were resolved and granulation tissue formed a clean wound bed. (D) Local skin flap coverage was performed after 12 days of wound care.

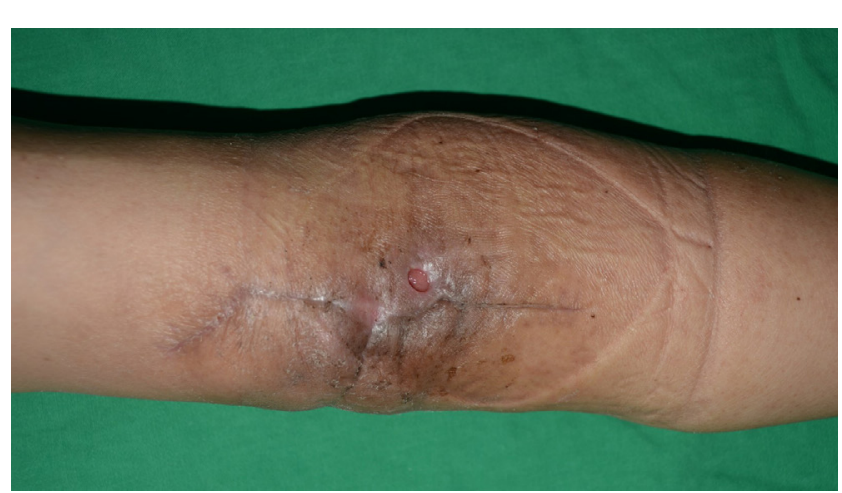

Fig. 4. Large seroma accumulation after first discharge. Repetitive accumulation of large seromas under the closed wound caused skin dehiscence.

\section{Discussion}

The major components of pesticides can be divided into three types, chlorinated hydrocarbons, acetylcholinesterase inhibi- tors, and botanical agents. Chlorinated hydrocarbons have been largely banned worldwide due to their long half-life which caused environmental pollution. Botanical agents, pyrethrum being one of the most commonly used, have lower toxicity and rarely cause systemic poisoning. Acetylcholinesterase inhibitors include organophosphates and carbamates. Organophosphates, mainly used in agriculture, inhibit choline esterase activity irreversibly. Carbamates are commonly found in household insecticides and are reversible cholinesterase inhibitors [1]. These agents accumulate acetylcholine at nerve synapses and cause both nicotinic and muscarinic symptoms such as excessive salivation, urination, nausea, vomiting, bronchorrhea, bronchoconstriction, skeletal muscle weakness, miosis, bradycardia and hypo- or hypertension [3-5]. The severity of symptoms varies depending on the amount, composition, and exposure route, and can lead to coma or death in severe cases. Unfortunately, it can be difficult to find out the exact type and amount of agent involved in intentional pesticide 

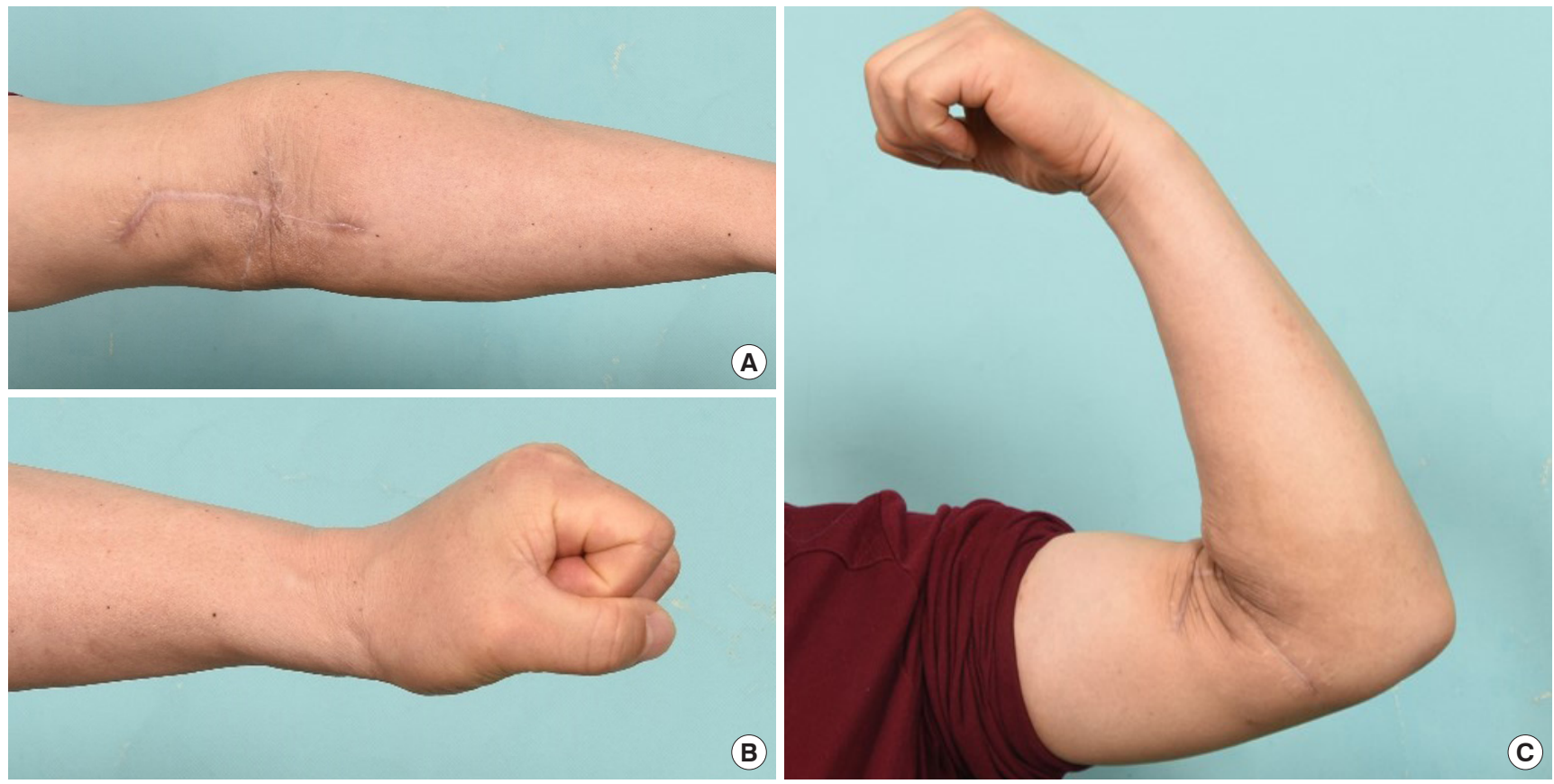

Fig. 5. Postoperative clinical view after 6 months. (A) The wound healed uneventfully after prolonged NPWT, revisional repair and compression dressings. No additional complications appeared within a 6 month follow-up period. (B, C) The patient presented full range of motion of left arm and hand with minimal strength and sensory limitation at 6 months.

poisonings. Thus, the patient's overall status should be checked initially and intensive medical treatment is needed early on. Intermediate syndrome can also occur in such cases, necessitating close observation during the recovery period [5].

Pesticide-induced local tissue damage should also be thoroughly treated for optimal functional results, especially in cases of extremity injection. Hydrocarbons mixed in pesticides dissolve lipid-rich membranes of the microvasculature and cause liquefaction necrosis. This results in clinical swelling, inflammation, cellulitis and necrosis of muscle, fat and skin. The affect can be similar to necrotizing fasciitis because these agents can spread along tissue planes and invade soft tissue [1]. This may increase inter-compartmental pressure resulting in severe sequelae [6]. These components are also not easily detoxified in local tissues; delayed intervention may prolong tissue exposure to the poisoning agents and exacerbate local and systemic damage. Therefore early surgical treatment including fasciotomy with serial debridement is required to remove toxins and contaminated tissue for successful wound management and prevention of late wound complications [1].

In this case, an unknown type and amount of pesticide was injected intravenously. Perhaps the patient's experience as a military corpsman helped facilitate accurate injection into the antecubital vein, as a result localizing tissue damage along the pathway of thrombosed and necrotic vessels, in return for relatively severe systemic toxicity including acute renal failure, hyperkalemia and mental changes. That surgical intervention was delayed for 9 days until after the patient's vital signs and blood chemistry were stabilized may have also exacerbated the toxic effects of the injected agent; brownish fluid with a strong pesticide smell had been retained in the wound area for this entire time span, and one phase of initial surgical debridement and NPWT was not sufficient to eliminate the pesticide odor and fluid. Only through serial debridement with NPWT could the toxins and liquefied tissue be eliminated, allowing clean granulation tissue formation and wound closure. Repetitive seroma formation with dehiscence also occurred after the surgical site had apparently healed, which was only successfully managed with prolonged additional NPWT and compression dressings; continuous observation was mandatory to ensure termination of treatment. Thankfully, no deep functional damage remained despite delayed intervention.

We hereby present a rare case of intravenous pesticide poisoning. Clinicians including the emergency and intensive care personnel who are generally in charge of initially managing such patients should recognize the actions of these agents which are poorly cleared in vivo and can cause abscess formation and liquifactory necrosis. Early surgical intervention with 
fasciotomy is necessary to remove the poisoning pesticides and limit systemic and local damage; serial debridement and NPWT are the norms for successful outcomes and functional restoration of the involved extremity. Furthermore, follow-up care should be done putting in mind that additional wound complications may occur even after apparent healing.

\section{Conflict of interest}

No potential conflicts of interest relevant to this article were reported.

\section{References}

1. Buchman MT. Upper extremity injection of household insecticide: a report of five cases. J Hand Surg Am 2000;25:764-7.

2. Guven M, Unluhizarci K, Goktas Z, et al. Intravenous or- ganophosphate injection: an unusual way of intoxication. Hum Exp Toxicol 1997;16:279-80.

3. Namba T, Nolte CT, Jackrel J, et al. Poisoning due to organophosphate insecticides. Acute and chronic manifestations. Am J Med 1971;50475-92.

4. Hise LL, Copeland R, Ortiz J. Injection of insecticide: a review of the literature. Orthopedics 1995;18:571-2.

5. Badhe A, Sudhakar S. An intravenous organophosphate poisoning with intermediate syndrome: an unusual way of intoxication. Indian J Crit Care Med 2006;10:191-2.

6. Shusterman EM, Williams SR, Childers BJ. Soft tissue injection of hydrocarbons: a case report and review of the literature. J Emerg Med 1999;17:64-5.

7. Hanane E, Mahmoud K, Saad EH, et al. Subcutaneous injection of organophosphate parathion: an unusual way of intentional acute poisoning. J Med Toxicol 2016;2. 